\title{
In-Situ Tritium Measurements Of The Tokamak Fusion Test Reactor Bumper Limiter Tiles Post D-T Operations
}

\author{
C.A.Gentile, C. H. Skinner, K. M. Young, M. Nishi ${ }^{1}$, S. Langish, S. O'hira ${ }^{1}$, A. von Halle, \\ D. Mueller, L. Ciebiera, D.Shaltis, T. Yamanishi ${ }^{1}$, T. Hayashi ${ }^{1}$ W. M. Shu ${ }^{1}$, A. Carpe, \\ K. Isobe ${ }^{1}$, E. Kearns, Y. Iwai ${ }^{1}$, C. Bunting, Y. Kawamura ${ }^{1}$
}

Princeton Plasma Physics Laboratory, Princeton University, Princeton, New Jersey, 08543 USA

${ }^{1}$ Japan Atomic Energy Research Institute, Tritium Engineering Laboratory, Tokai, Ibaraki 319-1195, Japan

\begin{abstract}
The Princeton Plasma Physics Laboratory (PPPL) Engineering and Research Staff in collaboration with members of the Japan Atomic Energy Research Institute (JAERI), Tritium Engineering Laboratory have commenced in-situ tritium measurements of the TFTR bumper limiter. The Tokamak Fusion Test Reactor (TFTR) operated with tritium from 1993 to 1997. During this time $\sim 53,000 \mathrm{Ci}$ of tritium was injected into the TFTR vacuum vessel. After the cessation of TFTR plasma operations in April 1997 an aggressive tritium cleanup campaign lasting $\sim 3$ months was initiated. The TFTR vacuum vessel was subjected to a regimen of glow discharge cleaning (GDC) and dry nitrogen and "moist air" purges. Currently 7,500 Ci of tritium remains in the vacuum vessel largely contained in the limiter tiles. The TFTR limiter is composed of 1,920 carbon tiles with an average weight of $\sim 600$ grams each. The location and distribution of tritium on the TFTR carbon tiles are of considerable interest. Future magnetically confined fusion devices employing carbon as a limiter material may be considerably constrained due to potentially large tritium inventories being tenaciously held on the surface of the tiles.
\end{abstract}

In-situ tritium measurements were conducted in TFTR bay L during August and November 1998. During the bay L measurement campaign open wall ion chambers and ultra thin thermoluminscent dosimeters (TLD) affixed to a boom and end effector were deployed into the vacuum vessel. The detectors were designed to make contact with the surface of the bumper limiter tile and to provide either real time (ion chamber) or passive (TLD) indication of the surface tritium concentration. The open wall ion chambers were positioned onto the surface of the tile in a manner which employed the surface of the tile as one of the walls of the chamber. The ion chambers, which are (electrically) gamma insensitive, were landed at four positions per tile. The geometry for landing the TLD's provided measurement at 24 positions per tile. The instrumentation was positioned on the tiles ( 96 tiles in each bay) by technicians who manipulated the boom and end effector from outside the vacuum vessel port. In addition to obtaining bay L tile measurements, 3 bumper limiter tiles were collected from the vacuum vessel for further analysis. During the bay L measurement campaign it was observed that several of the tiles in the lower third of the bumper limiter exhibited considerable surface flaking. 


\section{Introduction}

The Tokamak Fusion Test Reactor which is located at The Princeton Plasma Physics Laboratory in Princeton, New Jersey operated for the time period of 1983 through 1997. During the final four years of TFTR operations, 1993 to 1997 approximately 53, $000 \mathrm{Ci}$ of tritium was injected into the TFTR vacuum vessel mainly through neutral beam injection [1,2]. When TFTR finished operation in preparation for final shut down an aggressive three month campaign commenced for the purpose of recovering tritium inventory which was deposited on the internal surfaces of the $>80,000$ liter volume vacuum vessel [3]. The TFTR vacuum vessel tritium cleanup campaign included; baking out the vacuum vessel to $150{ }^{\circ} \mathrm{C}$, glow discharge cleaning with deuterium as the working gas (and later with a mixture of $10 \%$ oxygen in helium), purging the vacuum vessel with dry nitrogen at pressures from 20 to 600 torr, and "moist air" purging with $40 \%$ relative humidity air $[2,4]$. During the three month tritium cleanup campaign approximately $40 \mathrm{kCi}$ of tritium was recovered from the vacuum vessel. Following the vacuum vessel tritium cleanup campaign several vacuum vessel purges and vacuum vessel vents were performed in support of the in vessel surface tritium measurements. Two years after the TFTR vacuum vessel cleanup campaign approximately $7,500 \mathrm{Ci}$ of tritium still remains tenaciously held on the 1,920 carbon tiles and stainless steel interior of the vacuum vessel. It was known from earlier measurements of TFTR and other fusion devices during deuterium operations that carbon based limiter tiles have an affinity for accumulating hydrogen isotopes $[5,6]$. The accumulation of tritium onto the carbon limiter is facilitated by the formation of a co-deposited layer of tritium and carbon which becomes deposited at various depths on the surface of the tile depending on the location in the vacuum vessel.
Surface measurements of the distribution of tritium on the limiter tiles were needed to collect empirical data for developing a model for the accounting of tritium both in TFTR and in future fusion devices for tritium material control and accounting purposes. Accurate tritium accountability in tokamaks and other tritium burning fusion devices is critical in maintaining the in vessel tritium inventory to quantities which comply with site specific criteria and associated safety analysis. It was these reasons that motivated the need to perform surface tritium measurements of the TFTR limiter.

\section{Measurements Techniques}

The in-situ surface measurements of the bay L TFTR limiter tiles were collected to measure the tritium activity on the surface of the bumper limiter tiles. Technicians worked from outside the TFTR vacuum port with a remote handling boom and were able to "land" tritium surface detection equipment on the surface of limiter tiles mounted on the inner wall at the small major radius. Three measurement techniques were used to collect the tritium surface measurements. The first was the deployment of an open wall ion chamber which, when landed on the surface of the tile, completed the ion chamber geometry as the surface of the tile became the dynamic wall of the ion chamber. The ion chamber was effectively placed at four different locations on each tile. The second surface measurement technique used ultra thin (50 micron) thermoluminscent dosimeters which, when placed directly on the surface of the limiter tile, gave a strong (passive) signal, the result of tritium beta decay emanating from the surface of the tile. In addition the TLD's were positioned in a matrix holder such that 24 discrete locations could be measured for each tile in the limiter (Fig. 1). 


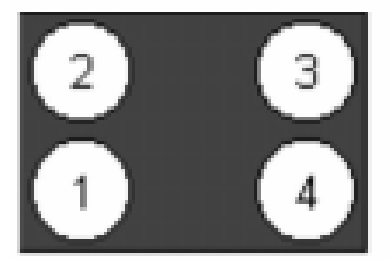

a)

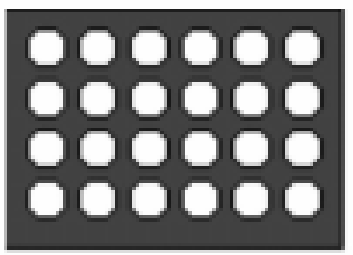

b)
Figure 1.

a) Ion chamber tile measurement locations

b )TLD tile measurement locations

The third surface measurement technique chosen was a pin diode employed as a solid state charged particle detector. The use of pin diodes to measure tritium surface contamination had been employed for similar purposes prior to the TFTR limiter measurements with success [7]. In the case of the TLD and pin diode tritium detection methods the active component of the detector needed to be physically placed on the surface of the tile to ensure that the relatively low energy tritium beta particles (E Beta max $18.6 \mathrm{keV}$ ) would be efficiently detected in an air atmosphere.

For the first set of bay L limiter tile measurements it was found that the employment of the open wall ion chamber resulted in the best technique for collecting data employing the remote boom and end effector (Fig. 2). Upon the opening of the TFTR vacuum vessel it was found that the interior of the vessel was coated with carbon dust. Radiological smear surveys of the dust indicated that the material was highly contaminated with tritium and ranged from 10 million dpm / $100 \mathrm{~cm}^{2}$ to 100 million $\mathrm{dpm} / 100 \mathrm{~cm}^{2}$. In addition the tritiated dust exhibited a static charge. Instruments placed inside the vacuum vessel with the remote position boom would become plated with dust and required extremely careful handling to ensure that the spread of tritium contamination did not occur outside of the TFTR vacuum vessel. Instrumentation remotely positioned into the vacuum vessel would within minutes become covered with the highly tritiated dust. The contamination of the in vessel measuring devices became further amplified when instrumentation contact was made with the limiter wall. Of the three techniques, the open wall ion chamber attached to the remote positioning boom was the least susceptible to the tritium-contaminated dust when it was placed on the surface of the carbon tiles, and that reproducible measurements were possible. TLD's placed on the surface of the carbon tiles became highly tritium contaminated and required many hours to decontaminate prior to analysis. The length of time required to remove tritiated dust from the TLD's masked the signal resulting from placing the TLD on the surface of the carbon tile for 10 minutes. The pin diode detector experienced similar tritium contamination issues and in addition is extremely sensitive to light. Positioning the detectors required a great deal of lighting for technicians as to ensure that the detector head was landed at the correct tile measurement coordinates. The open wall ion chamber exhibited only minor increases in background signals resulting from the plating out of tritiated dust on the face of the detector (background signals were subtracted during the measurement). In addition the detector was electrically configured to mask out the $\sim 45 \mathrm{mR} / \mathrm{hr}$ internal vacuum vessel gamma field which is the result of the activation of the steel, on which the tiles are mounted, during the 1993 to 1997 D-T campaign. As a result of its reliability in producing good quality data during this first entry into the vacuum vessel for performing the bay $L$ in situ measurements the first complete set of bay $\mathrm{L}$ tritium surface data were collected with the open wall ion chamber method. 


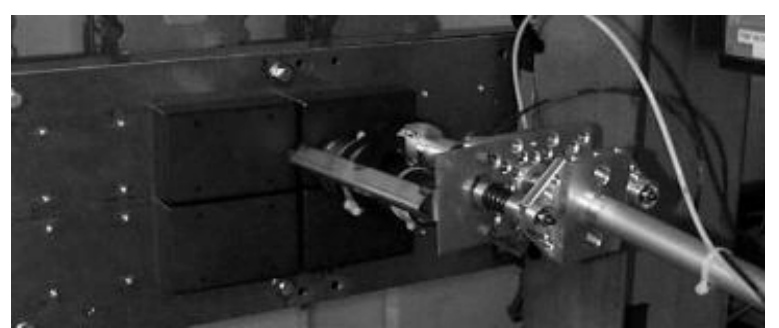

Figure 2. Remote boom and end-effector with open walled ion chamber

In addition to the tritiated carbon dust it was also observed that many of the tiles located in the bottom third of the TFTR limiter were flaking [8]. This phenomena had never been previously observed on the TFTR limiter tiles. The flakes are extremely fragile and have a tendency to fall off the face of the tiles with any mechanical agitation. The thin flakes ( $\sim 10-50$ microns thick) range in size from several $\mathrm{mm}$ to $\sim 1.5 \mathrm{~cm}$ in length and are ultra fragile (Fig. 3).

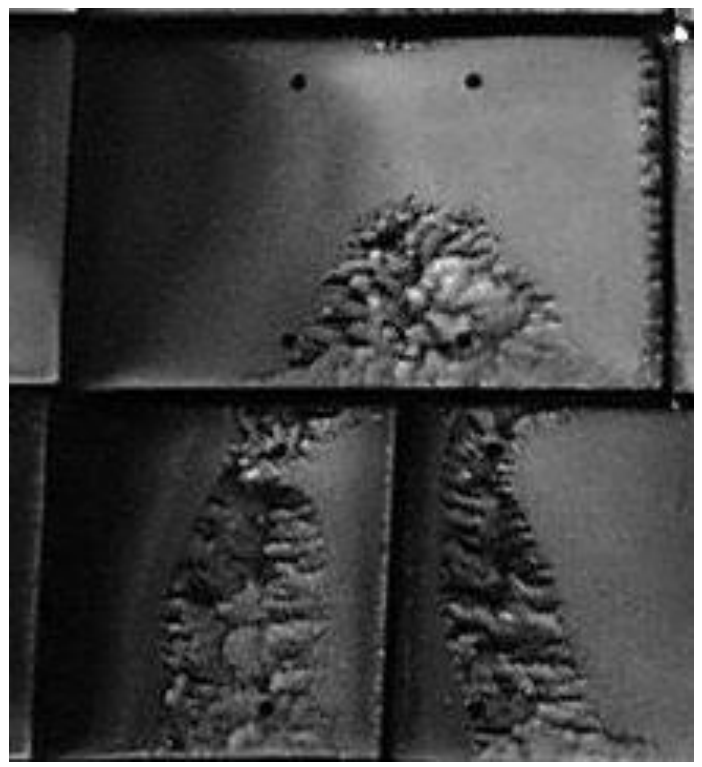

Figure 3. TFTR carbon limiter tile flaking

\section{Results}

The bay L limiter wall is composed of 96 carbon tiles configured in four columns with 24 tiles per column. Typical tile dimensions are $\sim 8 \mathrm{~cm}$ wide, $\sim 12 \mathrm{~cm}$ long, $\sim 3.5 \mathrm{~cm}$ deep with an average weight of approximately 600 grams per tile. During these measurements the open wall ion chamber was positioned at four locations per tile and data was collected from each of the measured locations. Surface measurements employing the open wall ion chamber ranged from $25 \mu \mathrm{Ci} / \mathrm{cm}^{2}$ to $400 \mu \mathrm{Ci} / \mathrm{cm}^{2}$ with the majority of the measurements ranging between $110 \mu \mathrm{Ci} / \mathrm{cm}^{2}$ to $220 \mu \mathrm{Ci}$ / $\mathrm{cm}^{2}$. The bay L limiter is missing 11 tiles in column $\mathrm{C}$, the result of an intervention during the summer of 1997 into the vacuum vessel, employing remote tools to collect bay L limiter tiles for external analysis. A graphical representation of the bay L tritium surface measurements is shown in figure 4 .

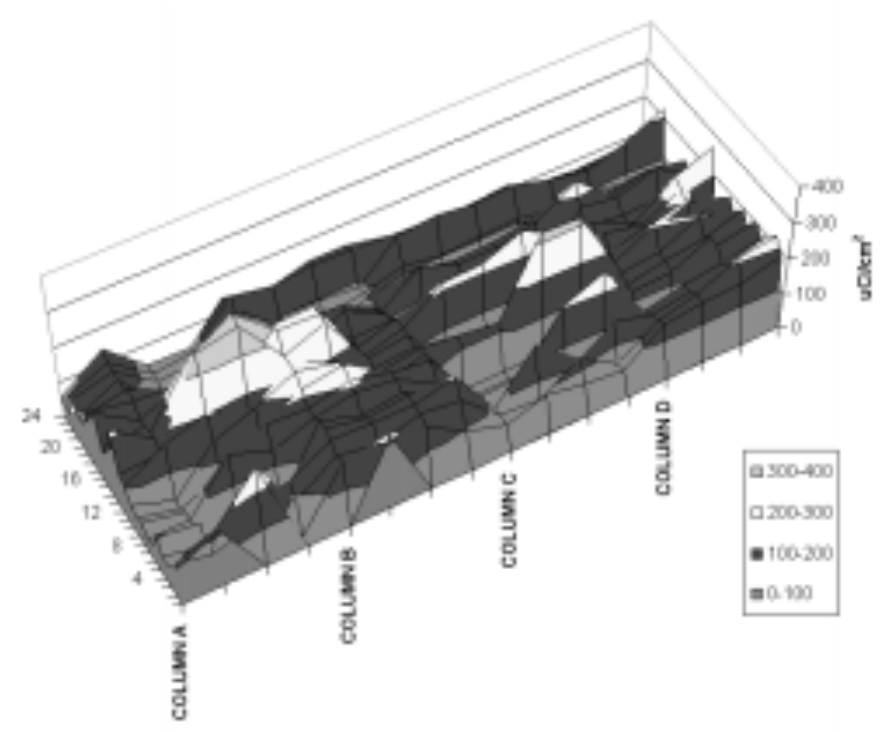

Figure 4. Bay L tritium surface measurements

External vessel tritium measurements for five of the bay L tiles and one flake were made employing a bake-out oven at temperatures of $350{ }^{\circ} \mathrm{C}$ and $500{ }^{\circ} \mathrm{C}$. The bake-out of the 5 bay L tiles supported the data collected during the in-situ bay L surface measurements which is that the distribution of tritium entrapped on the carbon limiter tiles in bay $\mathrm{L}$ is not homogenously distributed. Tritium recovered from the individual tiles ranged from $0.065 \mathrm{Ci}$ to $1.590 \mathrm{Ci}$. (Fig. 5.). The flake which weighed 0.24 grams released $0.74 \mathrm{Ci}$ of tritium during bake-out. It appears that a significant portion of the 
tritium contained in the carbon tile is entrapped in the surface flakes. Since the first observation of tile flaking (November 1998) in the bottom third of the bay $L$ limiter it has since been observed in other bays during later vacuum vessel openings. Currently the flaking is more pronounced and can be observed in the middle and top sections of the TFTR limiter tiles.

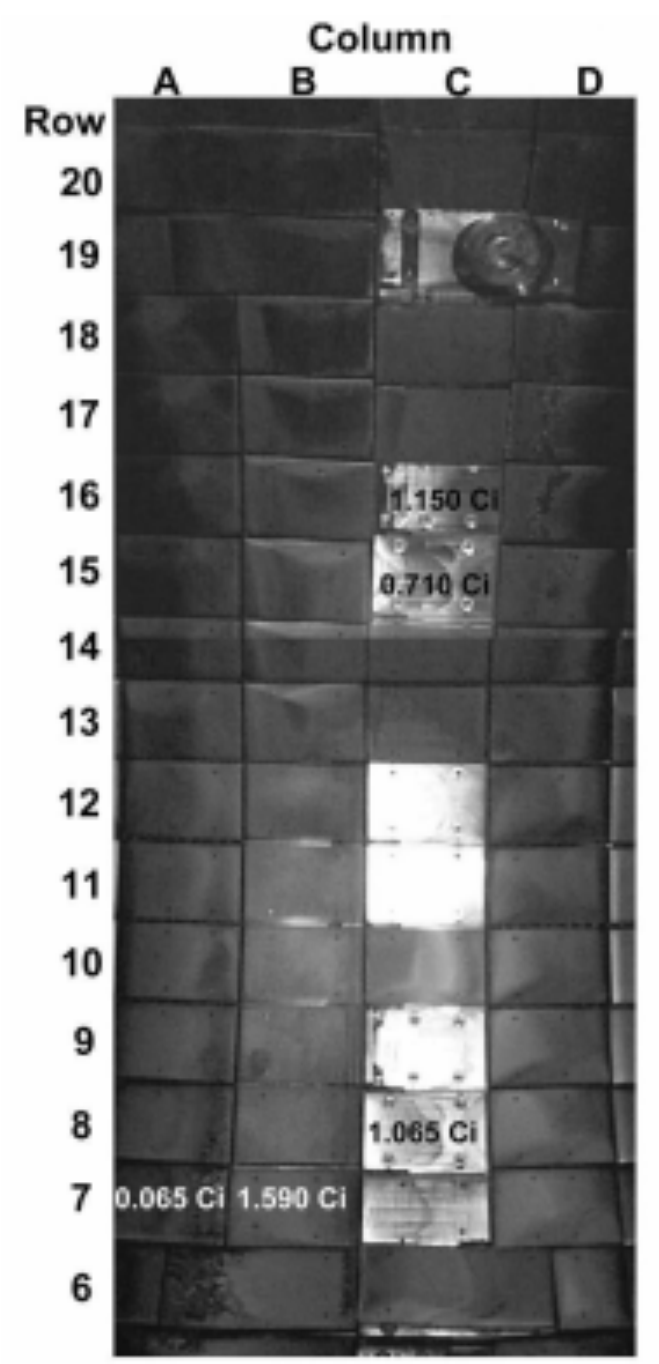

Figure 5. Curies removed from bay L tiles by bakeout

\section{Future Activities}

Future plans include people entering the TFTR vacuum vessel through the bay $\mathrm{K}$ port, employing positive pressure bubble suits for radiological protection against the tritiated carbon dust. Bay $\mathrm{K}$ is in pristine condition since there have been no remote tile removal or in situ measurement activities which might have disturbed the deposition of tritium on limiter tiles in this region of the TFTR vacuum vessel. The goal of the bay $\mathrm{K}$ entry will be to perform additional in situ tritium measurements with an enhanced pin diode detector head configuration to mitigate the effects of light and tritium dust contamination, a new slightly off set $(<0.5 \mathrm{~mm})$ TLD matrix holder to reduce the tritated dust plating to the TLD's, and open wall ion chambers. Measurements will be made of the limiter tiles and other surfaces (stainless steel) inside the vacuum vessel. In addition 41 bay $\mathrm{K}$ limiter tiles for external vessel analysis will be collected along with activation coupons which were fixed in TFTR prior to the use of tritium in the device. An attempt will be made to collect flakes (intact) and to recover other unidentified deposits (Fig. 6.) plated out on the lower section of the bay $\mathrm{K}$ limiter for external analysis. It is also planned to perform a comprehensive photographic survey of the inside of the vacuum vessel prior to the decommissioning of TFTR.

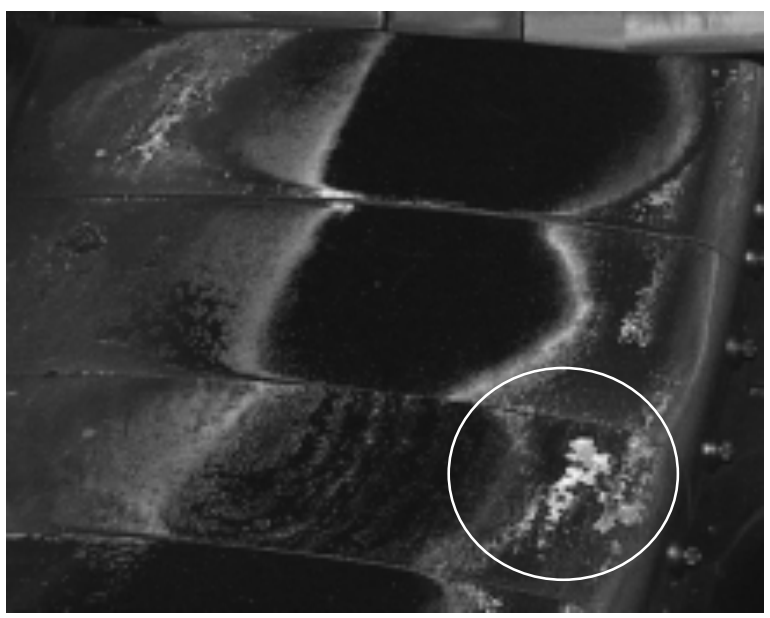

Figure 6. Unidentified deposits

\section{Conclusion}

Data obtained by the in-situ bay L tritium surface measurement and subsequent bakeout of the tiles indicate that the deposition of tritium in the TFTR vacuum vessel is not homogenously distributed. The formation of flakes on the surface of the 
limiter tiles have been analyzed and contain a relatively high concentration of tritium. Further in-situ and external vessel measurements and analysis are required to better understand the dynamics of the distribution of tritium in a magnetically confined tokamak configuration. Instrumentation employed for in-situ surface tritium measurements in carbon lined (D-T fusion) vacuum vessels need to be compatible with the highly tritium contaminated dust environment.

\section{Acknowledgments}

The authors would like to acknowledge the efforts of the PPPPL Health Physics Staff for safely and efficiently controlling radiological conditions associated with the in situ tritium measurements of the TFTR bay L limiter tiles. Funding for the in-situ measurements was provided by JAERI through the Annex IV to the JAERI / DOE Implementing Arrangement on Cooperation in Fusion Research and Development, and by USDOE Contract numbers DE-AC02-76CH03073 and DE-AC05-96OR22464.

\section{References}

[1] Gentile, C.A., Kalish, M., et al., "The Operation of The TFTR Tritium System" 17th IEEE/NPSS Symposium Fusion Engineering. Vol. 1, No. 97CB36131.

[2] Mueller, D., Blanchard, W., et al., "Tritium Retention and Removal on TFTR". 17th IEEE/NPSS Symposium Fusion Engineering. Vol. 1, No. 97CB36131.

[3] Nagy, A., Amerescu, E., et al., "Tritium Recovery from the TFTR Vessel" 17th IEEE/NPSS Symposium Fusion Engineering. Vol 1, No. 97CB36131.

[4] Hosea, J., et al "The Tritium Fuel Cycle on TFTR and Related Issues" Presented at IEA Workshop, Tritium Experience in Large Tokamaks; Application to ITER. Princeton
Plasma Physics Laboratory, Princeton, N.J. March 16 - 18, 1998 Nuclear Fusion, Vol. 39 page 271 (1999).

[5] Wampler, W.R., et al., "Deposition of carbon, deuterium, and metals on the wall and limiters of the Tokamak Fusion Test Reactor". Journal Vacuum Science Technology. A6, 2111-2115. (1998).

[6] Wampler, W.R., Doyle, B.L., Pontau, A.E., "Hydrogen and deuterium in graphite limiters from TFTR, PDX and PLT". Journal of Nuclear Materials. 145 - 147, 353 - 356. (1987).

[7] Wampler, W.R., Doyle, B.L., "Lowenergy beta spectroscopy using pin diodes to monitor tritium surface contamination". Nuclear Instruments and Methods in Physics Research. A 349473 - 480. (1994).

[8] Skinner, C.H., Gentile, C.A., Menon, M.M., Barry, R.E. "Flaking of co-deposited hydrogenated carbon layers on the TFTR limiter". Nuclear Fusion, Vol. 39, No. 9. (1999). 